\title{
Le sacrifice chez les Bambara et les Malinké
}

Sacrifice among the Bambara and Malinke

\section{Youssouf Cissé}

\section{CpenEdition}

\section{Journals}

Édition électronique

URL : https://journals.openedition.org/span/494

DOI : $10.4000 /$ span.494

ISSN : 2268-1558

\section{Éditeur}

École pratique des hautes études. Sciences humaines

\section{Édition imprimée}

Date de publication : 30 mai 1981

Pagination : 23-60

ISSN : 0294-7080

\section{Référence électronique}

Youssouf Cissé, «Le sacrifice chez les Bambara et les Malinké ». Systèmes de pensée en Afrique noire

[En ligne], 5 | 1981, mis en ligne le 04 juin 2013, consulté le 21 septembre 2021. URL : http://

journals.openedition.org/span/494 ; DOI : https://doi.org/10.4000/span.494 


\section{E S A C R I F I C E C C E Z I E S}

\section{B A M A R A E T LE S M A L I N K E}

par

Youssouf Cissé

I. Mises à mort et sacrifice primordial dans le mythe cosmogonique (1)

Au plus fort des cérémonies annuelles célébrées au niveau du Komo (2), c'est à dire au moment où l'on immole à tour de bras chiens et poulets sur les objets cultuels, les adeptes de cette société d'initiation murmurent à qui mieux mieux : "tout sacrifice est une "louange" (adressée) à Dieu (une glorification de Dieu), à plus forte raison un sacrifice sur les autels dyoo" (3). Par sacrifice, sonni, il faut entendre ici l'ensemble des gestes et attitudes qui précèdent, accompagnent et suivent 1'immolation des victimes, celle-ci donnant son nom à la cérémonie tout entière. C'est en tout cas ce que disent les responsables mêmes du Komo pour qui le sonni est par excellence 1 'acte par lequel dyòn ni seen fiza,

(1) Les matériaux rapportés dans le présent article ont été tirés des récits initiatiques fondamentaux du Komo. Ces récits, appelés kow sinti, kow dyudyòn ou kow dyun baxi tyoko, sont détenus par les maîtres dyalenfa qui ne les enseignent qu'aux initiés de leur choix. Cet enseignement se fait presque toujours en privé, pour ne pas dire en secret.

(2) On trouvera une description relativement complète de ces cérémonies dans G. Dieterlen et Y. Cissé, Les fondements de la société d'initiation du Komo, Paris-La Haye, Mouton et Cie, pp 251-286.

(3) sonni bè ye ma tanu ye dyo sòn bè ye ma tanu ye.

Text. :

"Tout sacrifice est une louange à Dieu,

Tout sacrifice à un dyo (autel dressé) est une louange à Dieu" 
"le petit être à deux pieds" (qu'est 1'homme) purifie son coeur (sòón) à l'égard de Dieu, se rabaisse et se soumet (sơơn) à lui avant de demander ses faveurs ou implorer sa grâce. On retiendra tout de suite ici que, dans la langue mandingue, le mot sònni, qui désigne le sacrifice, signifie à la fois "arrosage", don, offrande du coeur et soumission, de même que les mots sơn, sờn et sòon (des homophones presque) signifient arroser, donner, se soumettre et coeur (4).

Les mêmes dignitaires du Komo ajoutent qu'il n'est point d'entreprise digne de ce nom pour la réussite de laquelle le Bambara ou le Malinké ne ferait au moins un sacrifice, celui-ci étant le plus souvent prescrit par un devin (5) et précédé, accompagné ou suivi, selon les cas, de formulation de voeux pieux, de récitations de prières, etc. (6). Ainsi, pour défricher un nouveau champ, ouvrir une mine, creuser les fondations d'une maison, entreprendre des démarches matrimoniales ou un voyage, aller à la chasse, introniser un chef, enterrer un mort ou célébrer ses funérailles, initier de jeunes gens, passer des examens, s'attirer les faveurs d'un supérieur, etc., les Bambara et les Malinké consulteront des voyants et feront les sacrifices, dons, offrandes, libations ... qui s'imposent.

Selon la nature de la victime en cause, les circonstances dans lesquelles il est fait, le lieu de son exêcution, le but qu'il vise, etc., un sacrifice est dit bon (sòni nyuman) ou mauvais (sòni dyugu).

(4) Nous verrons plus loin 1'importance capitale, au niveau du sacrifice, des rapports entre ces mots. Mais notons d'ores et déjà que les Malinké et les Bambara ont, dans l'enseignement initiatique, recours à 1 'homophonie, I'homonymie, I'analogie, etc., pour montrer la connexion des choses ici-bas.

(5) Même au niveau des sociétês d'initiation, le sacrifice est toujours précédé de la divination; la preuve en est ce cantique du Komo :

"Vieux fauve à la tête fêlée (parce que) blanchie par la science! I1 faut (lors des cérémonies du Komo) pratiquer la voyance (1a divina-

Avant de faire les sacrifices ..... tion)

Faire les prières

Et faire acte de soumission

Afin que les initiés du Komo soient sauvés par toi."

Cf. G. Dieterlen et Y. Cissé, op.cit., p. 261.

(6) Nous donnerons et analyserons plus loin les prières, ainsi que les termes malinké-bambara employés au niveau des différents types de sacrifices. 
Lorsqu'un chef de famille immole par exemple un coq blanc (7) sur la tombe de son ancêtre pour lui demander de "se tenir debout" afin que ses descendants ne connaissent ni la faim ni la honte, il fait sans doute 1à un sacrifice bon. En revanche, enterrer vivant un âne noir après l'avoir muselé et en proférant des injures ou des incantations constitue un sacrifice mauvais dans la mesure où ce genre de mise à mort vise toujours à faire périr un rival qu'on aurait auparavant fait bâillonner magiquement.

Quel que soit leur genre, les différents sacrifices effectués par les Malinké et les Bambara ne seraient, selon une croyance solidement établie chez les responsables des cultes de ces peuples, qu'une simple répétition ou, comme le veut l'expression mandingue, "un retour sur" les sacrifices (ou les actes de mise à mort considérés comme tels) que Dieu lui-même aurait réalisés au commencement de la vie sur la terre et ceci dans le but soit de châtier les blasphémateurs, soit de sauver son oeuvre, en l'occurence l'édifice cosmique, soit encore de défendre ses adorateurs ou de préserver les valeurs spirituelles et morales qu'il leur enseigna de l'action des profanateurs. Ces "sacrifices" seraient au nombre de quatre (8) ; toutefois, un d'entre eux est dit sònni yèrè yèrè, "1e véritable sacrifice", comne si les autres n'avaient pas été effectués selon les règles de l'art.

Bref, avant d'examiner lesdits sacrifices, résumons très brièvement les évènements qui les ont précédés et qui aideront sûrement à les comprendre.

(7) Nous verrons quelle importance ont la robe, la couleur et le teint dans le sacrifice chez les Malinké et les Bambara,

(8) S'agit-il là d'un chiffre symbolique? Il y a tout lieu de le croire, car, comme nous le verrons plus loin, il existe une grande variété de sacrifices. 
1. Eléments de cosmologie malinké-bambara (9).

Au tout commencement, I'univers, dunya ou dinyè, n'était que fir (10), un $f u$ de ténèbres, fu dibizen, un fu calme et sans mouvement, $f u$ madalen, un $f u$ frais (sans chaleur et sans vie), fu sumalen, un $f u$ lourd (dense), fu giviman. C'est alors qu'ayant décidé de créer l'univers, "le Mâ̂tre incréé et le Maître sans limite et sans fin, le Mâ̂tre qui n'a pas de bouche et qui parle"...(11) le fit au moyen des signes qu'il avait conçus dans le "mystère de son intérieur", a kónòna gundo (12).

Dieu fit tout d'abord introduire dans le fu "l'éclat, le bris,

(9) La genèse du monde selon les Malinkë et les Bambara est enseignée à travers des récits fort longs dans lesquels chaque étape importante de la création est représentée par un signe au moins. De nombreux et savants commentaires et exégèses ont trait aux noms de ces étapes et aux signes qui les représentent. Nous n'aborderons pas ici ces aspects du sujet qui seront traitês dans notre thèse de Doctorat d'Etat, intitulée "Les fondements mythiques et religieux des civilisations mandingues. En revanche, nous tenterons d'évoquer tout aspect de 1 a genèse du monde qui pourrait éclairer nos propos sur le sacrifice. La plupart des étapes de la genèse que nous évoquerons ici ont été traitées dans : G. Dieterlen et Y. Cissé, op. cit., pp. 63-213.

(10) Par fu, il faudrait entendre ici "la matière primordiale, inanimée et sans vie", même si le mot est couramment utilisé pour désigner le "rien", le zêro, le néant (cf. Pl. I).

(11) Lorsqu'ils enseignent l'histoire de la création, les mâ̂tres initiateurs du Komo déclament ici les 12 ou 14 "premiers grands noms" de Dieu qui sont : 1) masa danbali, "Dieu l'incrée" ; 2) et 3) masa dan tan, "Dieu sans fin et sans limite" et aussi "Dieu sans bouche". (masa da ntan), da tè masa min fè n'a bi kuma, "le Dieu qui n'a pas de bouche et qui parle" ; 4) madanmantan, "1e Dieu Eterne1" ; 5) fèn bè dyuu, "1e fondement de toute chose" ; 6) fèn bè kunn, "1a raison de toute chose" ; 7) fèn bè daminè, "le commencement de toute chose" ; 8) fèn bè Zaban, "la fin, le terme de toute chose"; 9) fèn bè dyudyón, "l'origine de toute chose" ; 10) fèn bè sinti, "1'appui, le support de toute chose" ; 11) fèn bè $b a$, "le substratum de toute chose" ; 12) fèn bè fa, "1e contenant, le contenu, le plein de toute chose" ; 13) so fla facma, "1e Puissant des deux mondes" ; 14) kanu masa kantòróna, "le Dieu d'amour et de peines".

(12) C'est ainsi, dit une leçon du Komo, que "I'univers, la vie, reste toujours un mystère, un mystère insondable" (dunya ye gundo ye, gundo dómbati; dunya ye gundo ye, kundo kóróbali). 


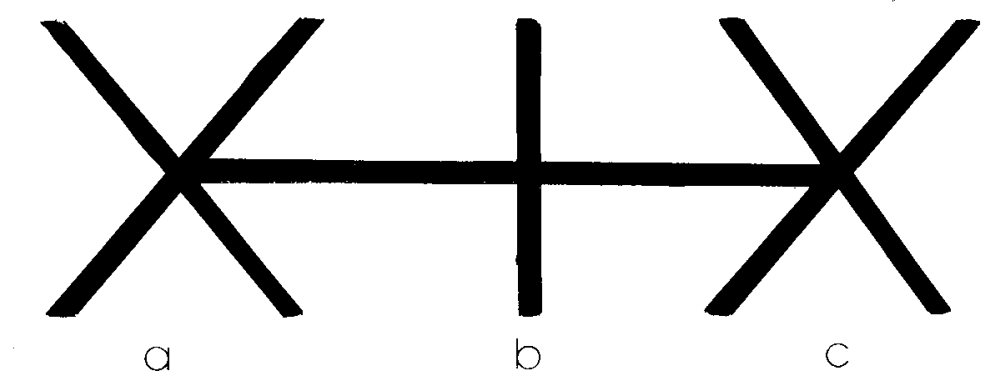

Pl-I_fu tì (Lesigne du fu)

Les trois croix composant le signe fu sont appelêes:

a) daminè (commencement); soròn ou suran tǐ (signe de l'êmergence sousentendu des mondes et des temps); bangi tí (le signe de la révélation et de la naissance); bugun ti (le signe de la multiplication des choses et des êtres); etc.

b) tyèmantyè (le juste milieu); dyŏ tí (le signe de l'édification sousentendu des mondes, des êtres et des choses); bazo ti (le signe de la vie); etc.

c) Zaban (la fin, le terme); sonsón tí (le signe de la convergence sousentendu des mondes et des choses vers un point minuscule où ils disparaîtront à jamais); etc.

Les branches de chacune des trois croix ci-dessus mentionnées représentent les quatre éléments fondamentaux (l'air opposé au feu, l'eau opposée à la terre) et leur point de jonction, appelé kisè "grain" et "pouvoir, puissance du signe", préfigure le nin, "le principe de vie" des êtres. 
le signe primordial", ti fòlò (13). Celui-ci produisit successivement l'éclair initial, yézéngu fólò, aussitôt suivi de la première vibration, yèrè-yèrè fòló, qui mit en mouvement, Zamaḱ, le fu. Ce mouvement était accompagné de la voix divine, mankon, dont devait pour l'éternité résonner l'univers. La crêation étant un processus continu et sans fin, on dit que cette vibration, qui se fait par cercles concentriques (14) tout comme la voix qui 1'accompagne, poursuit encore sa progression à travers le fu (1'incréé ?) et qu'il en sera ainsi jusqu'à ce que Dieu y mette fin et ceci par"sa volonté et son désir"(15) contenus dans le kara ou "esprit divin" qui circonscrirait le monde. C'est ce qui fait dire que dunya, le monde (ou la vie), qui est en soi "illimité", insondable, est seulement la partie du fu animée et éclairée par le kara (16). D'ailleurs, comme celui-ci, il est souvent matérialisé par un cercle appelé dunya kónòna ou dunya kònò bara, "le dedans, l'intérieur du monde" ou "la gourde, la sphère (matérialisant) l'intérieur du monde".

Dieu fit ensuite naître ou introduire successivement dans

(13) Ce signe primordial est également appelé ti kelen pe, "seul et unique signe", ti kalaman, "signe ardent, chaud", danni ni danbaa ka kelennya, "l'unité ou 1 'unicité du créateur et de la création", etc. (cf. G. Dieterlen et Y. Cissé, op. cit., p. 63 et p. 203). On dit que depuis 1 'introduction de ce signe dans le néant, 1 'univers ne connâ̂t point "le calme".

(14) Cette vibration se propagerait à la manière des cercles concentriques que $l$ 'on crée en jetant un caillou dans l'eau d'un étang.

(15) sanko ni dunkò. Mais ces deux mots signifient, par analogie, "l'affaire du cie1", san ko, et "l'affaire des profondeurs", dun ko.

(16) Cette conception du monde est loin de faire l'unanimité. Pour certains, Dieu aurait une fois pour toutes crée l'univers dans lequel les choses et les êtres naissent et disparaissent continuellement ; ce qui rend illusoire toute notion de fu existant au-delà de l'esprit créateur. Pour d'autres, il faudrait entendre par monde seulement ce qui peut être apprêhendé, perçu et imaginê par les sens et 1 'esprit humain. Le reste relève du domaine de Dieu, c'est à dire du fu. En revanche, tout le monde est d'acoord pour affirmer que la vie est née de la chaleur, elle-même née du mouvement dont le symbole par excellence est le cercle, kara, ce qui revient à dire que la vie est 1 'oeuvre de Dieu et de lui seul. Nous exposerons ces différentes théories dans notre ouvrage sur les civilisations mandingues. 


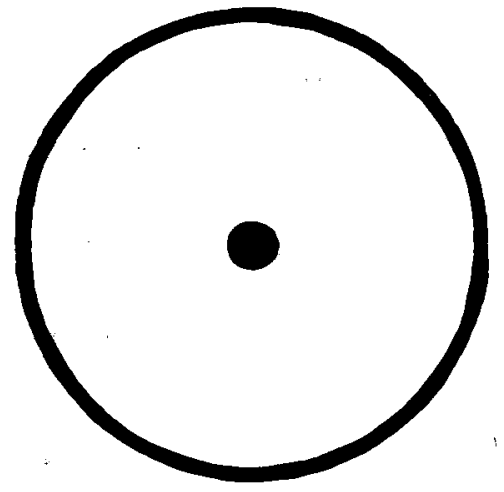

Pl_ I I kara_

"cercle parfait", esprit ardent.

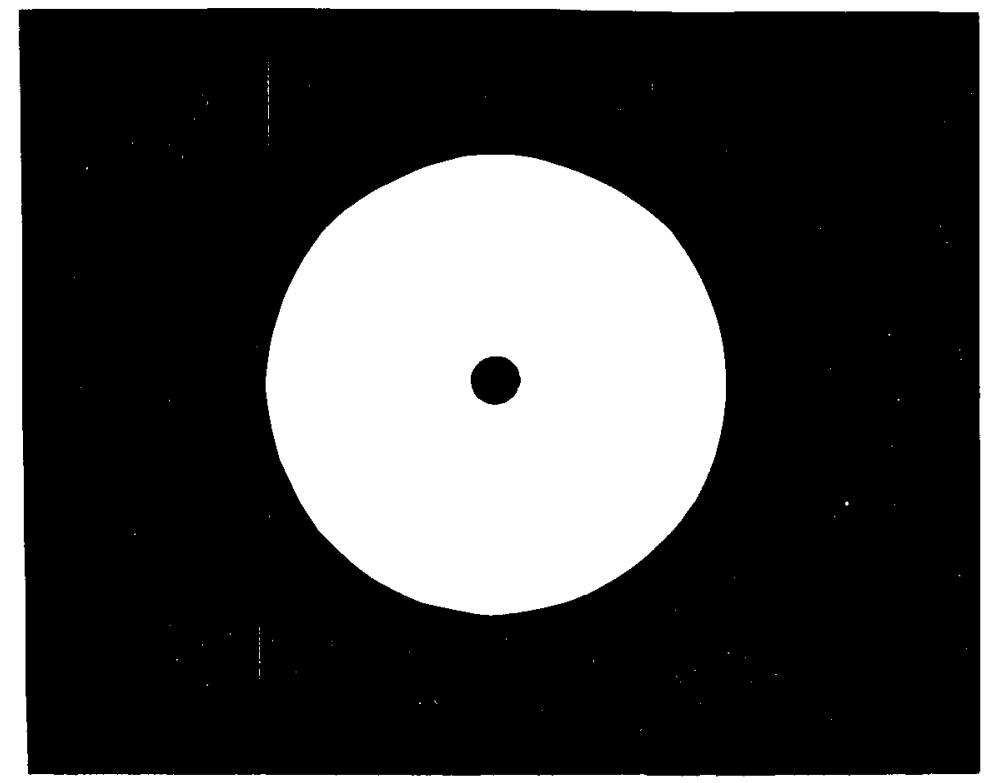

dyèn kōnòna

dyèn konona: "le dedans de l'univers" et "le dedans de la vie". Au-delà du cercle qui le délimite et en-deçà du point qu'il contient, dyèn ou dunya est inaccessible.

"Le monde est un mystère, un mystère inconnaissable;

la vie est un mystère, un mystère insondable"

(Leçon bambara) 
1 'univers ainsi créé un certain nombre "d'ensembles graphiques structurés" (17) dont la fonction sera d'imprimer au monde les "marques divines", taa ma suèn, et d'y perpétuer à jamais la "volonté et le dẻsir du créateur".

Le premier de ces ensembles graphiques introduits dans le monde fut le "signe du tournoiement", mumun ti, lui-même issu du ti kelen pe (18). Le monun ti engendra notamment le glan zo, puis le glan glan zo qui, pareils à des mouvements browniens, brassèrent, malaxèrent, entrelacèrent le contenu de l'univers.

L'action du glan głan zo aboutit notamment à la rëvêlation, bangi, des"grands signes de la création", danni ti bow (19), qui constitueront la base de la création de tout ce qui va exister dans les temps et les mondes (les cieux, les terres, les choses, les êtres animés, etc.) ; et ils auront comme témoins, combien éloquents et éclatants, dans $1^{\prime} u^{-}$ nivers les étoiles, doolo (astres, planètes et constellations), et surtout la voie lactée (20), symbole de la prolifération future des signes de base (21), comme de celle de la vie qui naîtra de ces signes (22). Pour parachever la création du monde et régler pour toujours ses mouvements, édifier ses structures, fixer son destin et "rendre

(17) On trouve dans les quelques planches que nous reproduisons dans cet article et dans : G. Dieterlen et Y. Cissé, op. cit., p. 178 et suivantes et p. 200 et suivantes, les plus importantes de ces graphies.

(18) Cf. supra p.28 note 13 et infra P1. III

(19) Il est à noter que le mot bow signifie aussi "mères", "sacrês" "substrats". A propos de ce mot, une leçon du Komo dit : dunya sigilen ti bow de ma, "1e monde est "assis", repose, existe grâce aux grands signes". Ces derniers portent par ailleurs le nom de manti, "signes divins" (cf. G. Dieterlen et Y. Cissé, op. cit., p. 309).

(20) Pour les Malinké et les Bambara; Ia voie lactée, appelée doolo sira ba, "la grande voie des étoiles", ou nyugun-nyugun ba, le grand plissement, contient dans les profondeurs de ses couches l'étoile de chaque être.

(21) On compare la multiplication de ces signes à celle des ce1lules soko kisè, "les grains de chair" à partir d'un oeuf de poisson ou d'une ovule fécondée, dite dyoli kuru. "boule de sang".

(22) En créant les "grands signes", Dieu aurait en même temps crée les nin, les "principes de vie" des êtres (cf. infra, p.33) 


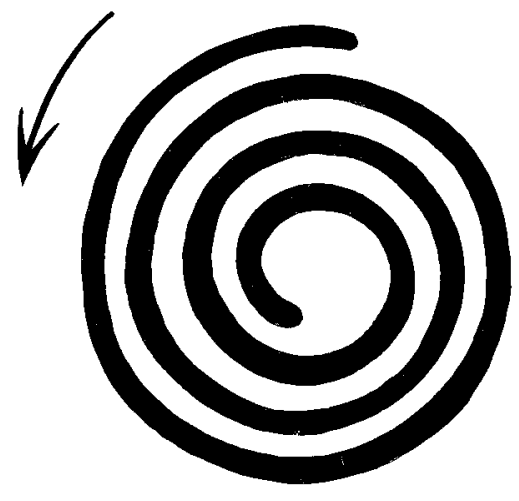

lasirili tr

(signe de la fécondation)

Pl I II. munun tri

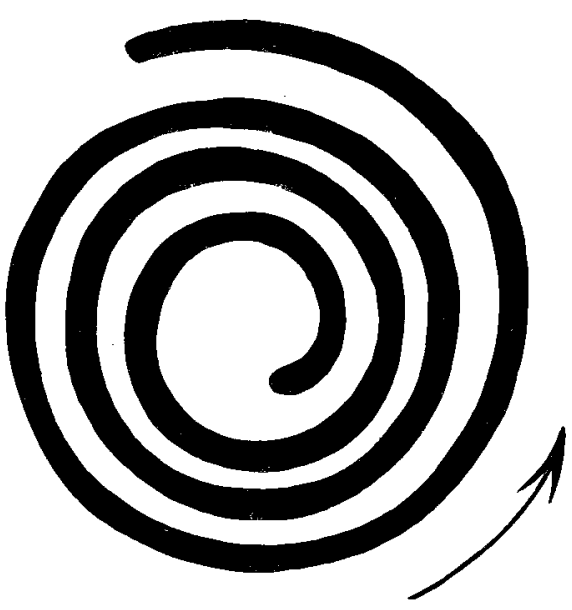

labilali tí (signe de 1a "libération")

("signe du tournoiement") 
nette", embellir son harmonie, Dieu fit introduire dans l'univers les graphies suivantes :

-- ma dyigin ni ma yèlèn, "descente de Dieu et montée de Dieu" (P1. IV).

Il engendrera d'une part le mouvement perpétuel de vie qui va du Créateur vers ses crêatures et des créatures vers leur Créateur, et d'autre part l'ascension des "âmes" après la mort et le mouvement ascendant et descendant du nyoma, "force vitale" (bonne ou mauvaise), des ancêtres et des victimes sacrificielles immolées sur les autels dyo;

-- suman ngolo (23), "N'Golo (mâ̂tre) de I'humidité", garant du maintien de 1 'humidité dans 1 'univers et de l'écoulement des eaux dans l'espace et sur la terre ;

-- waran kana (24) ou waka ti (25) ou dunya fankan nyan (26), mainteneur de 1 'harmonie du monde, signe régulateur du déroulement des temps et de la rotation universelle (P1.V);

- banan ngolo (27), "N'Golo (mâ̂tre) des conceptions et des enfantements" (Pl.VI). Cette graphie aux connotations multiples est pour ainsi dire le sceau que Dieu apposa à l'univers au terme de sa création; et ce sceau est à l'image de la personne, mòkò, comme si le Créateur voulait à tout jamais signifier que la personne est et restera la raison et le but ultime de son oeuvre.

(23) Cf. G. Dieterlen et Y, Cissê, op. cit., p. 179, et Y. Cissé, "Signes graphiques, représentations, concepts et tests relatifs à 1 a personne chez les Malinké et les Bambara đu Mali", in La notion de personne en Afrique noire, ed. G. Dieterlen, Paris, CNRS, 1973, p. 139 (et suivantes) et p. 169 (et suivantes).

(24) De waran, disperser, dilater à 1 'extrême, et kama, maître du ciel empyrée, waron kama signifierait "1'extension extrême de 1 'esprit divin" et désigne en tout cas l'univers créé dans son immensité.

(25) De waka, écartement, et de ti, signe ; waka ti désigne ici le temps, la durée des temps et des mondes.

(26) Text. : "irradiation, dêploiement de la force du monde".

(27) Cf. G. Dieterlen et Y. Cissé, op. cit., p. 200 (et suivantes) et $\mathrm{Y}$. Cissé, in La notion de personne ..., p. 139 (et suivantes) et $\mathrm{p}$. 174 (et suivantes). 
2. Les premiers êtres créés par Dieu.

Après qu'il eut fini "d'asseoir" le monde, man ngala (28) devait décider de "prendre la terre", de réaliser dans la matière les êtres vivants, nin ma fènw, qui peupleraient 1 'univers et, plus particulièrement, ceux d'entre eux pour 1 'amour de qui il créa le monde : les hommes ou, plus exactement, les personnes, mökòw (29).

Comme il a été dit plus haut, les nin ou "principes de vie" ou âmes de tous les êtres vivants furent conçus dès le commencement et en même temps, d'autant qu'ils découlaient des signes fondamentaux de la création ( $(30)$. C'est du reste pourquoi on dit : "un nin n'est pas plus âgé qu'un autre nin, même s'il est vrai qu'un nin apparâ̂t toujours à l'air (à la vie organique) avant un autre nin"(31). Et comme les signes, les nin sont considêrés comme intangibles (32).

Bref, Dieu devait tout d'abord créer dans le ciel un premier couple de jumeaux mixtes, Mousso Koroni et son cadet Pemba (33). A ces deux premières personnes qu'il créa belles, intelligentes, ardentes et puissantes, il devait non seulement faire part de ses desseins, mais aussi transmettre 1 'essentiel de ses connaissances, excepté celles permettant de crêer des êtres vivants ou de disposer de leurs nin, de prédire 1 'avenir ou de changer le cours du monde. Mais un jour qu'il leur parlait de l'amour, kanu, et de ses vertus, Mousso Koroni

(28) man ngala ou masa ngala, "le Maître (qui réside dans le) bleu bleu indigo (du ciel).

(29) CE. PI. VII

(30) Cf. supra, p. 28-30

(31) nin de bi bò finyè na nin nyè, n'o tè, nin man kòrò ni nin ye. Nombreuses sont les réflexions de ce genre sur le nin ; ainsi, nin bèè nin, "toute âme est une âme", c'est à dire, toute âme en vaut une autre et mérite autant qu'elle respect et protection.

(32) "Toute chose vieillit ici-bas, excepté le nin" : fèn bl bi kord dunya na yan, fo n'a kèra nin ye.

(33) Ces personnalitês mythiques portent selon les temps et les circonstances des noms bien précis : ainsi, Mousso Koroni, "la petite vieille femme" s'appelle selon les cas Nyâlen ou Nyakalen, Keleya, Farima, Tyènégèré, Dyinè Bilé, Daliko, Moussoni Dyinè Fâtoulé, Fourétou, Dyobali, etc. Quant à Pemba, on 1 'appelle aussi Tyèni, Tléko, Dyinè Massa, Dan Massa, etc. Elles sont respectivement représentées dans le ciel par le scorpion et la croix du sud. 


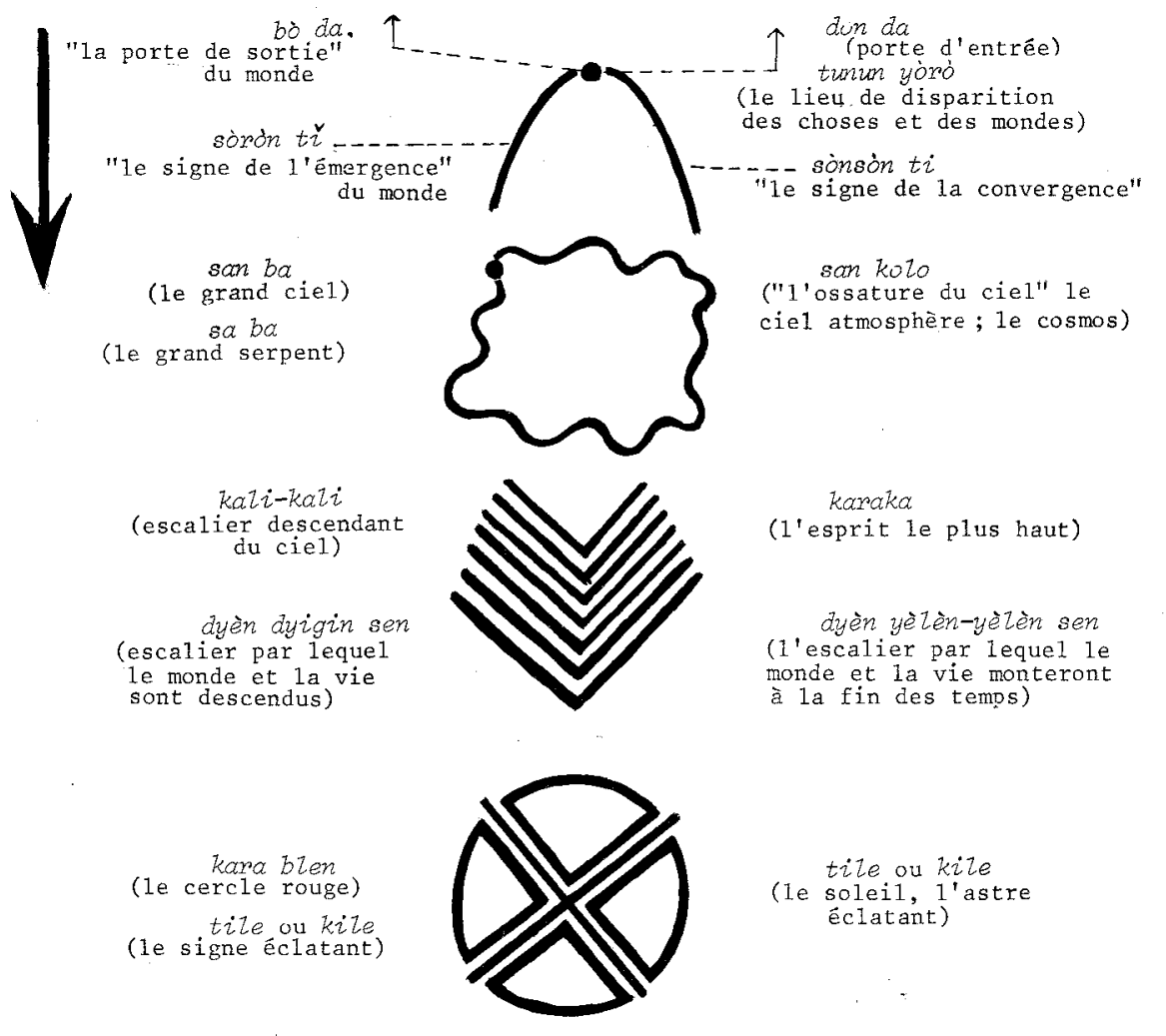

$d y i$ so

(la maison de 1 'eau) nyon so (la maison de la la vie)
respiration, de la

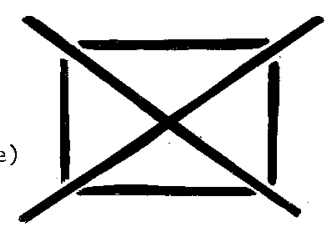

dyèn so

(la maison du monde.

le monde terrestre,

la vie d'ici-bas)

$d y i$ dooto

(1'êtoile de l'eau)

nyon kisè

(le pain de la vie)

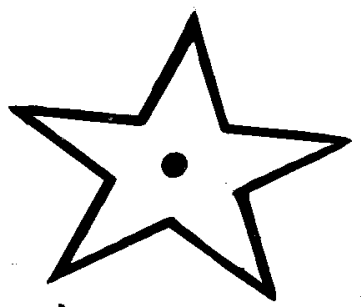

mókò doolo

(1'étoile de la personne)

nyd 2007o

(1'étoile du mili)

(1a porte d'entrée du monde)-.. Do_ bo da "porte de sortie" de la vie) bangi yoro (lieu de naissance)

Pl.IV. ma dyigin ni ma yelen 
PLANCHE IV

MA. DYIGIN NI MA YELEN

(Descente de Dieu (de 1'esprit de Dieu) et remontée de l'esprit de Dieu).

Cette graphie se lit de haut en bas et de bas en haut. Elle est censée montrer comment le monde et la vie sont nés et comment ils disparaîtront à la fin des temps.

Notons que l'expression masa dyigin ("descente du Mâ̂tre du Ciel") désigne chez les Malinké l'ensemble des récits de la création, des récits mythiques, légendaires et religieux plus certains grands récits historiques. 
(6)

(2)

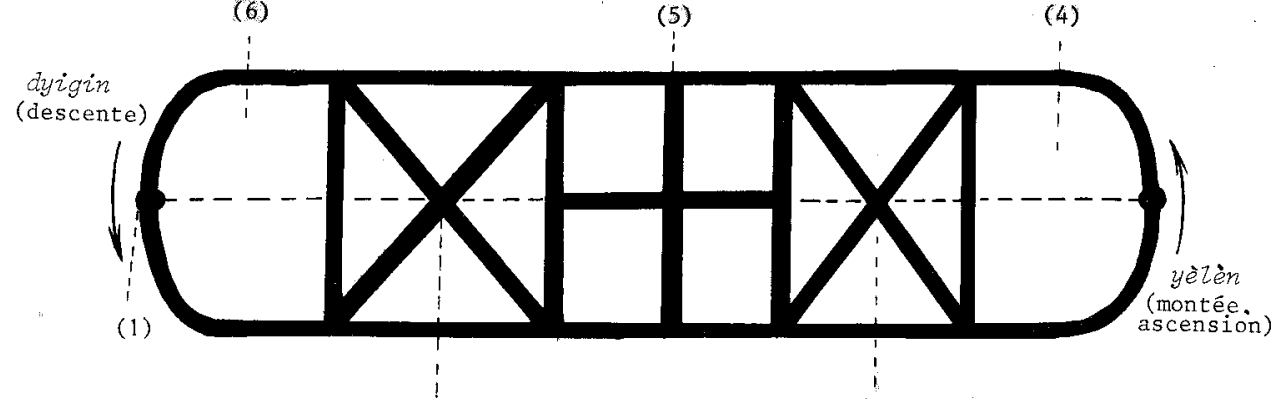

(4)

(3)

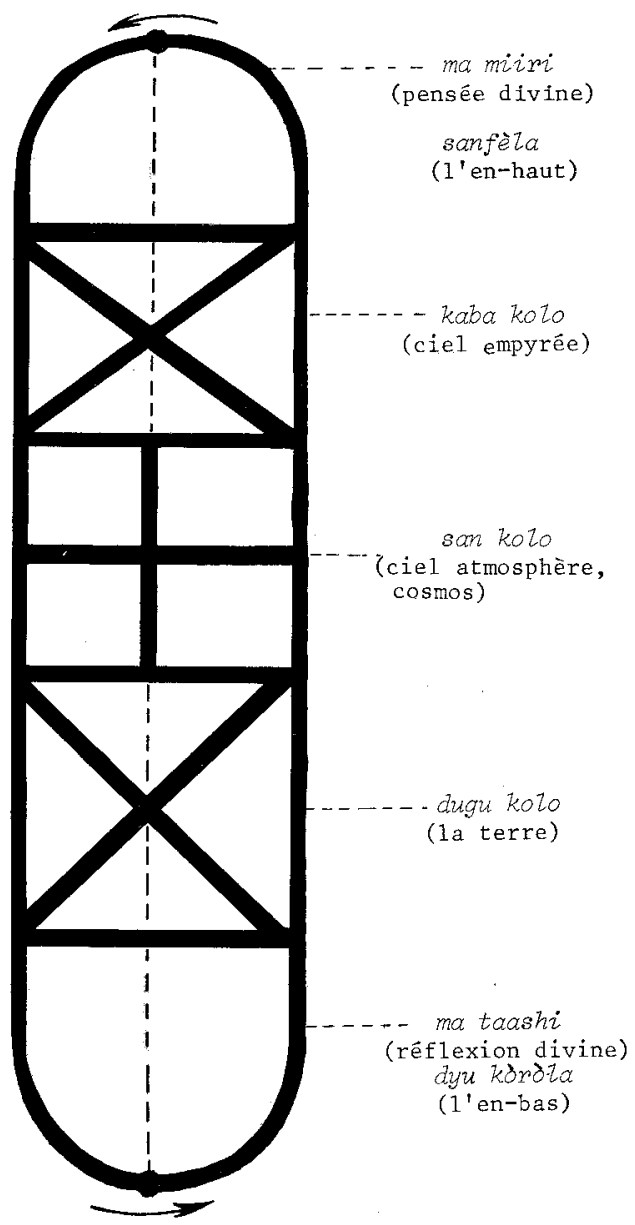

Pl.V_ Waran kama 
PLANCHE V

WARAN KAMA

(considêré horizontalement et verticàlement)

(les pointillés allant d'un pôle à 1'autre de la graphie matêrialisent 1'itinéraire suivi par tout être ou tout objet durant sa vie. Cet itinéraire est dit ç̉jèn Zatikè, "la traversée du monde".

(1) bó da (porte, lieu de sortie)

(2) dyèn so folo fan tan ni fiza (les 12 parties du premier monde, c.à d. le monde de Mousso Koroni)

(3) dyèn so flonan fan tan ni fiza (les 12 parties du deuxième monde -celui d'après le déluge- autrement dit le monde de Fâro).

(4) tunun so (la maison de la disparition) ou saya so (la maison de la mort).

(5) dyi ba so fan tan ni fila (les 12 parties de la maison des "grandes eaux", le déluge).

(6) bangi so (Ia maison de la révélation) ou dyigin so (maison des enfantements). 


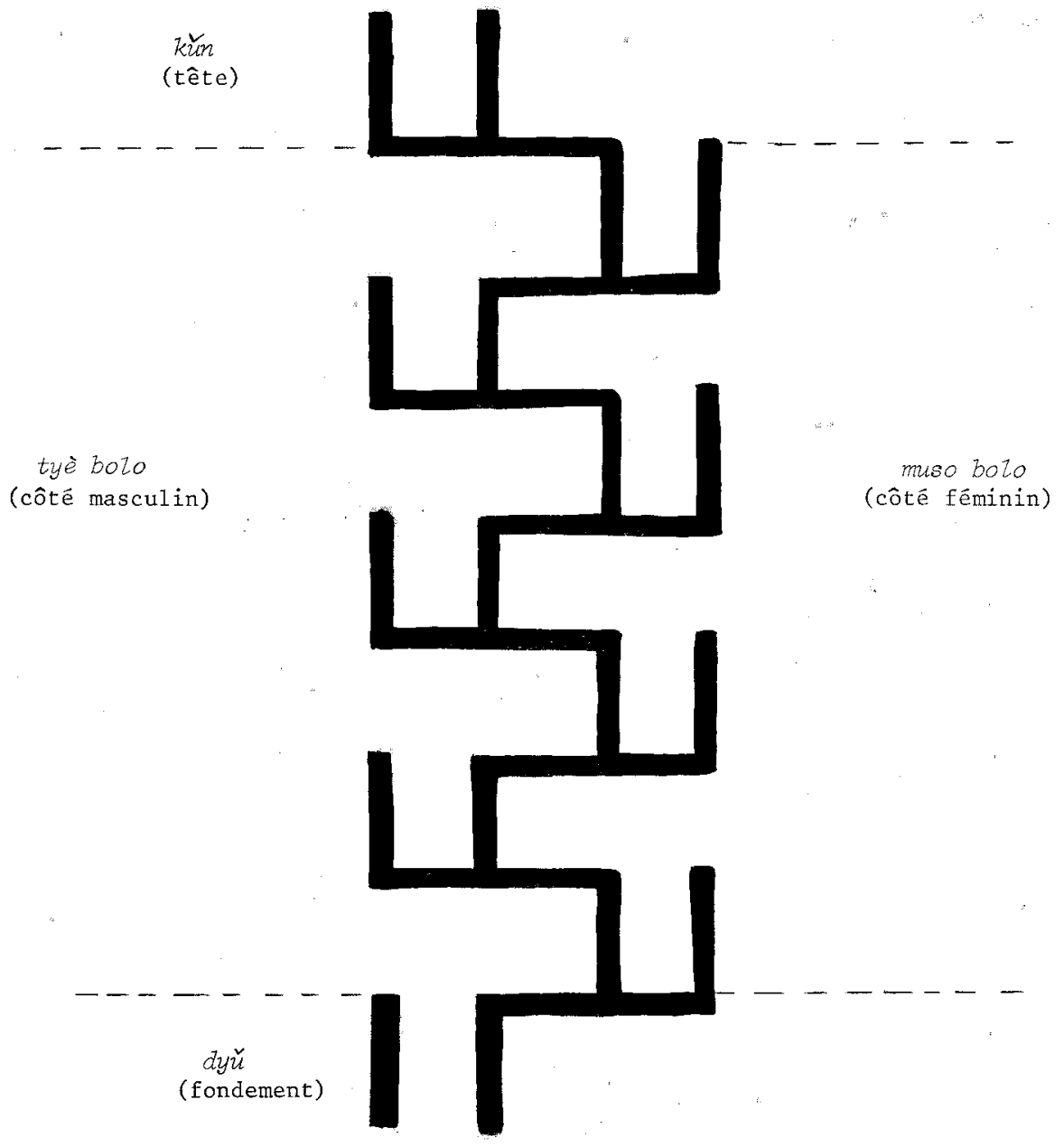

Pl_VI_Le banan ngolo.

Les 33 segments de la graphie symbolisent 1 'agercement des 33 éléments osseux de la colonne vertébrale, et le segment isolé l'os du pubis. Chaque étage de la graphie représente un des neuf mois lunaires que dure la gestation de l'être humain, soit 266 jours. 


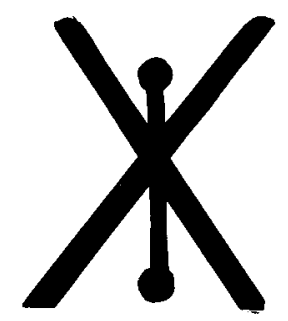

mòkò

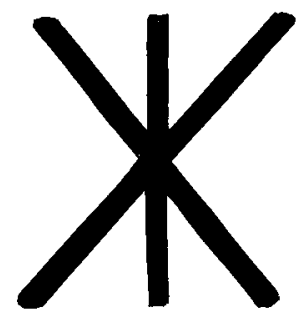

kòmò

(1a personne) - Pl-VII_ (la femme)

ici l'homme

Ce signe de la personne est celui que le créateur reproduira en procédant à la répartition des morceaux du bélier qu'il va sacrifier au ciel. 
l'interrompit et 1 ui demanda à brûle-pourpoint : "De tout ce que tu as créé, la mort, la vie, le ciel, la terre, les étoiles, le soleil, la lune, l'amitiê, la suprématie (mansaya), le savoir, la droiture, la croyance, la foi, la haine, l'amour, l'homme, la femme, la beauté, la grâce ... à quelle chose va ta préférence ?" (34) Devant le silence du Créateur, Mousso Koroni, hors d'elle-même et vociférant des paroles graves, sauta dans le cosmos, san kolo, après l'y avoir poussé son jumeau Pemba. Dans leur chute vertigineuse, ils perdirent connaissance ; ils ne virent par conséquent rien des espaces intersidéraux dont la connaissance véritable, ainsi que celle de leurs mystères, leur restera à jamais interdite. Ils ne revinrent à eux-mêtmes qu'au voisinage immédiat de la terre et ceci au moment où le cordon ombilical, bara dyuru, avait fini de se dérouler. Après plusieurs balancements et tournoiements fort désagréables et douloureux, leur lien avec le créateur cassa (35) ; ils tombèrent alors lourdement sur les talons, puis sur le coccyx, ce qui aura ultérieurement pour conséquence de déformer leur colonne vertébrale et de pervertir leurs "caractères", dyogo (36).

Très vite remise de sa commotion, Mousso Koroni va sans plus attendre amener son jumeau Pemba à commettre, après elle, à l'égard du Créateur Iui-même (et plus tard, à l'égard des messagers du Créateur sur la terre) les plus grands sacrilèges qu'aucune créature ait jamais commis à l'égard de Dieu (37). Ainsi, pour signifier leur rupture avec le Créateur, le deux jumeaux dansèrent, quelques instants

(34) C'est depuis ces temps-1à, dit-on, qu'une véritable confusion des sentiments trouble toujours les rapports entre la fille aînée et son père.

(35) Dieu aurait ainsi privé Mousso Koroni et Pemba de leur placenta et de leur cordon ombilical, ce qui fera d'eux des êtres instables, insatisfaits et insolents, des êtres à la dérive. On les appelle du reste maa sim tikèzen,"les gens dont les attaches furent rompues", sous-entendu avec leur créateur.

(36) Pour les Bambara et les Malinké, toute déviation importante de 1 a colonne vertébrale entraîne fatalement une déviation psychologique ou sexuelle, ou les deux.

(37) Les plus grands de ces sacrilèges sont évoqués dans les pages qui suivent. 
seulement après et à 1 'endroit même de leur chute, une danse obscène en vociférant ce chant :

$$
\begin{aligned}
& \text { n'ta ye } \\
& \text { n'ta ye } \\
& \text { n'ta ye } \\
& \text { n'kerra n'yèrè ta yè } \\
& \text { kuu kukuy (39) }
\end{aligned}
$$

Trad. :

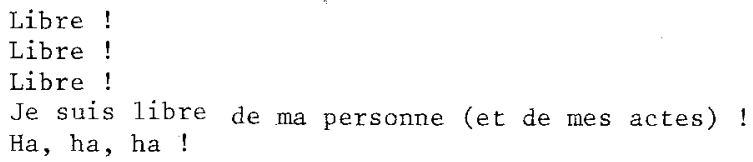

Dès Iors, les propos et les actes de Mousso Koroni et de ses futurs partisans appelés muso kòròni denw, "les enfants de Mousso Koroni", vont être d'une violence et d'une obscénité telles qu'elles mettront en péril l'oeuvre divine. Et c'est pour sauvegarder celle-ci que le Créateur entreprendra les "sacrifices" dont il fut question plus haut et dont les motivations et les modalités nous sont fournies par le mythe que nous rapportons ci-dessous.

\section{Premier sacrifice fait par Dieu.}

Le premier des quatre sacrifices faits par Dieu fut la destruction massive des "gêants" après que ceux-ci eurent causé du tort au Créateur et porté atteinte à l'intégrité et à l'harmonie de la création, son oeuvre. Qui êtaient ces géants capables de nuire à Dieu ? C'est ce que nous apprend le fragment de mythe que nous allons résumer (40).

(38) Text. : "Ma propriété !

Ma propriété !

Ma propriêté!

Je suis devenue la propriété de moi-même. Ha, ha, ha !"

(39) Rire sarcastique à l'adresse de Dieu.

(40) Ce mythe, dont nous ne livrons ici que quelques passages très succints, est appelé muso kòròni ka kow, "les choses, les affaires", les actes de Mousso Koroni, ou encore muso kòròni ti ie kow, "les êvènements du "soleil", de l'ère de Mousso Koroni". Il tient, aux dires mêmes des initiés bambara, une place de choix dans les prolégomènes du savoir traditionnel dans la mesure où il traite de la personne humaine considérée dans tous les aspects de son être et de sa vie. 
Rouges écarlates, comme le tissu murufe blen (41), et grands au point que leurs têtes se perdaient dans le siel, les dyamandyan, "les grands de la brousse inculte" ou les dyinè dyan fólów, "les premiers grands génies", furent, après Mousso Koroni et son jumeau Pemba dont ils sont (indirectement) issus, les premières personnes à avoir vếcu sur la terre. En effet, pour diminuer la puissance de ces deux personnages qui venaient de le quitter à la suite d'un différent né de la "jalousie", Dieu fit arracher leur arbre (42) et le fit précipiter de 1 'en-haut vers 1'en-bas. En tombant sur la terre, ledit arbre, qui s'était presque complètement consumé -- il avait pris feu et était devenu incandescent dans sa chute -- vola en éclats, à l'exception de son coeur qui deviendra le penpele ou penbele (43). Plus tard, chaque éclat, chaque débris de ce qui restait de 1 'arbre de Mousso Koroni et de Pemba se transforma et devint par clivage une paire de jumeaux mixtes. Les personnes qui virent ainsi le jour grandirent avec le temps et devinrent des géants dont la taille était telle qu'il leur suffisait de lever le bras pour s'emparer des étoiles et les offrir comme jouets aux "enfants" (44).

Tous les faits et gestes de ces géants étaient autant de dêfis. lancés à Dieu, car ils avaient épousé la cause de Mousso Koroni et de son jumeau qui n'arrêtaient pas, depuis leur descente sur la terre, de blasphémer et même de nier toute suprématie du Créateur sur les choses et les êtres.

Les choses en restèrent là jusqu'au jour où, excédé par les

(41) Selon M. Delafosse (La Zangue mandingue et ses dialectes, Vo1. 2, Paris, Imprimerie Nationale, 1955, p. 524), le mot mumfe wuze désignerait un tissu de drap de couleur rouge êcarlate.

(42) Chacun des deux couples de jumeaux primordiaux avait son arbre au ciel. Celui de Mousso Koroni et Pemba fut anéanti. Quant à celui de Fâro et Bemba, appelé san toro, "le figuier du ciel", il serait matérialisé par le toit conique des sanctuaires : ses feuilles seraient les chaumes et ses racines, la pointe du toit.

(43) Cf. infra, p. 52

(44) Il faut entendre ici par "enfants" les plus petits des géants. Etant nés d'éclats de différentes dimensions, ils n'avaient par conséquent pas la même taille. 
sarcasmes de Mousso Koroni et les gestes de ses enfants (45), Dieu fit entrer le ciel en collision avec la terre (46). Ce fut 1 'hécatombe chez nos géants qui périrent en grand nombre, volatilisés, ou disparurent, enfoncés dans la terre où ils restent à jamais prisonniers (47). En dépit de la violence et de l'étendue du désastre, i1 y eut des rescapés parmi les géants, mais ceux-ci ne mesuraient plus qu'une coudée et un empan au maximum, comprimés, compacts, condensés, ratatinés qu'ils étaient sous la violence du choc; d'oì les différents noms sous lesquels ils sont connus.

\section{Deuxième sacrifice fait par Dieu.}

La deuxième mise à mort effectuée par Dieu au début des temps est appelée sònni yèrè yèrè, "le vrai sacrifice". Comme nous le dit la suite du mythe ci-dessus évoqué, il s'agissait de celui d'un bêlier, destiné à purifier et à renforcer l'édifice cosmique construit par Dieu et qui était sur le point de s'écrouler.

Seuls Mousso Koroni et Pemba sortirent indemnes du cataclysme. En effet, instruits par Dieu, alors qu'ils vivaient auprès de lui dans le ciel, des signes annonciateurs de la collision entre le ciel et la terre, ils s'étaient, le moment venu, réfugiés dans un vestibule rocheux qu'ils avaient depuis longcemps repéré au fond d'une va1lêe encaissée.

Une fois la catastrophe passêe, Mousso Koroni et Pemba, loin de désarmer, n'éprouvaient que plus de ressentiment et de haine pour leur

(45) Ceux-ci, dit-on, voulaient crever le plafond du ciel empyrëe derrière lequel Dieu se tiendrait, selon eux, à $1^{\prime}$ abri du regard de ses crétaures.

(46) Les commentaires relatifs à plusieurs des 266 signes du Komo font état de ladite collision (cf. G. Dieterlen et Y. Cissé, op. cit., pp. 126, 128 et 134 , et les signes $n^{\circ} 79,83,85,87$ et 104 pour ne citer que ceux-1à). On dit à propos de cet évènement que "le Créateur fit retentir en Mousso Koroni et ses enfants le tambour destructeur de leurs signes spécifiques" donbaa ma ye tatinyè dunun fò $u(a)$.

(47) Les plus grands des géants se seraient volatilisés ; les moyens furent enfoncés dans la terre et seraient devenus les mangusi (déformation de mango si, "la race des réprouvés de Dieu") ; les plus petits seraient devenus les nains vivant sur la terre. 
créateur ; ils allaient en cela jusqu'à s'interdire de prononcer son nom et de regarder en direction du ciel pour ne pas avoir 1'air de 1 'implorer. Et ils n'arrêtaient surtout pas de le critiquer en clamant à son adresse de "fortes paroles", kuma bow, des paroles fortes comme celles-1à : "Seuls ceux qui doutent d'eux-mêmes et craignent pour leur pouvoir se tiennent dans les ténèbres" ; "quand on prétend s'appeler kanuba, "Mère Amour" ou "Celui qui aime ou qui est amour" (48), on ne massacre point des innocents, d'autant que "1'amour ne sait tuer et encore moins assassiner" (49).

Excédé par le silence (pour ne pas dire l'indifférence) de Dieu, Mousso Koroni, devenue presque folle, décida alors de s'attaquer à trois domaines dont Dieu disait qu'ils constituaient le badyu, "le fondement mère", l'essence de la création. Mousso Koroni eut d'abord des rapports sexuels avec son jumeau, enfreignant ainsi ce que danbaa ma, le "Mâ̂tre Créateur", avait ordonné : "ne vous cherchez pas tant que je ne vous dirai pas de le faire et n'ayez jamais de rapports contre nature; et sachez que de même que la chance réside dans la tête, de même 1 'amour vêritable réside dans l'âme" (50). E1le ne tarda d'ailleurs pas à se lasser des prestations de son jumeau qu'elle quitta sans prévenir pour allẹ de par le monde à la recherche de plaisirs plus enivrants (51), plus pervers, plus douloureux. Elle fit ainsi 1'amour avec la terre, le tronc des arbres, les épines, le vent, les rayons de soleil ardents, les cailloux, les fourmis rouges, bref avec tout ce qui pouvait, en l'écorchant, la brûlant ou lui faisant

(48) Les noms et attributs de Dieu sont fort nombreux chez les Bambara et les Malinké. La société d.'initiation en retient 266 , appelés "les grands noms du créateur".

(49) kanu tè fèn faka, sanko $k^{\prime} a$ dyu fa. Text. : "l'amour ne tue pas une chose et encore moins l'assassine".

(50) kunnadiza bè kun de rò, diyanya bè nin de rò.

Ces deux vers constituent le thème d'un très beau chant d'amour connu des peuples mandingues et intitulé nin na, "dans 1'âme".

(51) dyarabi ye dòtò le di, "1'amour est comme une boisson fermentée", disent les Malinké. 
mal, calmer son appétit (52). Màis plus elle s'acharnait à assouvir son désir, plus elle devenait folle de désirs (53). Il en était de même pour Pemba qui, en l'absence de sa jumelle, fit tout ce qu'il pouvait faire pour jouir lui aussi.

Après qu'elle eut parcouru le monde tout entier, Mousso Koroni se retrouva un beau jour à son point de départ, avec ses problèmes (54). Mais partout où elle êtait passée sur la terre toute vie avait disparu et ceci sous 1 'effet conjugué du nyoma (55) qui émanait de son

(52) Ces fornications de Mousso Koroni et surtout 1 'onanisme qu'elle pratiqua en tous points de la terre sont évoqués ou imités au cours de certains rituels. Ainsi, pour critiquer les femmes qui refusent de leur donner un peu de céréales ou la menue monnaie lors de la quête qu'ils font annuellement, les jeunes gens de la compagnie du wara demanderont au porteur de masque de leur groupe de se coucher à plat ventre sur le sol et d'exécuter les mouvements du coìt, et ceci au rythme des battements de mains et de ce chant exécuté par tous ses compagnons :

miso wo

$n^{\prime} i$ ma wizi

Trad.:

dontòni satò ye yan

Eh! femme

Si tu ne te réveilles pas

La petite bossue va mourir ici, (à ta porte).

(53) Pour les Bambara et les Malinké, les nymphomanes, surtout lorsqu'elles sont très belles -- et elles le sont le plus souvent -sont considérées comme étant 1 'incarnation de Mousso Koroni.

Et selon la sentence, "qui épouse une femme étonnante (de beautê), verra dans sa "suite" ou en elle au moins une des trois choses suivantes :

-- des hommes étonnamment fortunés, ou forts, ou beaux, ou laids, ou des gueux, etc. ;

-- des maux de ventre étonnamment douloureux (tels que règles douloureuses, grossesses nerveuses et autres maladies gynécologiques compliquées ) ;

-- des génies étonnamment puissants" (qui s'empareront de son corps et de son être et feront d'elle une pervertie sexuelle et une aliénée mentale).

(i ma na dakabana muso fum, i bè dakabana fèn saba nin dò, i b'o y'a ko : n'i ma dakabana tyèw $y^{\prime} a$ kò, i bè dakabana kònò dimi y'a $2 a$, walima dakabana dyinè.)

(54) De très beaux chants de jeunes filles et de mariage retracent ce retour de Mousso Koroni. corps,

(55) Force vitale émanant du nin ou principe de vie qui anime le 
être et du waanzo (56) qui. s'échappait de son sexe. Seuls des bazanzan (57), ces arbres bizarres qui, contrairement aux autres plantes, reverdissent en saison sèche et perdent leurs feuilles pendant la saison des pluies, avaient poussé aux endroits où elle avait pénétré la terre de son clitoris.

Bref, en retrouvant son jumeau, Mousso Koroni n'eut qu'une idée : avoir coûte que coûte des "enfants vrais", normaux. Elle se souvint alors de ces autres paroles de Dieu : "Tout rapport sexuel, pour être "prospère", fécond, devra être précédé, chez les couples, des opérations de la circoncision et de l'excision" (58). Mousso Koroni va s'attaquer à ce second domaine réservé de Dieu ("Faire de l'homme un homme et de la femme une femme n'est-il pas un acte divin ?"). Dans une crise de folie, elle coupera avec les dents le prépuce de son jumeau endormi et, avec ses ongles acérés, son propre clitoris. Effectuẻes dans la précipitation et sans les instruments adéquats (59), ces opérations eurent pour résultat d'enfler démesurément le gland de Pemba et de rendre hypersensible ce qui restait du clitoris de Mousso Koroni, interdisant ainsi tout rapport sexuel entre les deux jumeaux dont l'appétit ne cessait d'augmenter au fil des jours. De ce fait,

(56) Force obscure et dangereuse liée au prépuce et au clítoris et dont il faut débarasser les individus par la circoncision et l'excision.

(57) Acacia albida.

(58) Ces opérations portent les noms suivants :

-- bolokoli, "lavage, purification du membre" (viril sous-enten$\mathrm{du}$; ;

-- kènè-kènèzi, "rendre doublement sain, dynamique, viril" ;

-- sigi nègè kòrò, "placer sous le fer" (sous 1'action purificatrice le sexe des adolescents) ;

-- salidyi, "ablutions rituelles".

(59) Quand viendra l'ère de Fâro, les hommes et les femmes seront, au début, circoncis et excisées de deux manières :

-- Ies forgerons et forgeronnes seront au cours de leur sommeil opérés par les génies (dyinè) sortis du fleuve. (De telles opérations, pour le moins inexplicables, auraient lieu de nos jours encore et sont appelées ala bolokoli, "circoncision par Allah", ou dyinè bolokoli, "circoncision par les gënies")

-- les autres personnes par les feuilles d'une plante aquatique appelêe kdò mumu, le "couteau de la mare" ; et plus tard, à 1'aide de "pierres de foudre". 
le malheureux couple se sépara : alors que Mousso Koroni passait tout son temps en compagnie d'un gros chien qu'elle avait apprivoisé en le nourrissant de gros os, Pemba quant à lui ne cessait de courir après une ânesse (60) qu'il finit du reste par attacher à un balanzan. De leurs rapports avec ces animaux naquirent deux monstres : 1a chauvesouris, être qui n'est ni chien ni oiseau, et le bilisi (61), sorte de démon à grosses têtes multiples qui n'est ni homme ni bête.

Au fur et à mesure que Mousso Koroni et Pemba commettaient leurs actes (62), 1'odeur qui s'en dégageait empestait l'atmosphère en s'élevant, et elle était sur le point de "gâter", corrompre, détruire 1 'univers lorsqu'elle parvint jusqu'à Dieu. Celui-ci, pour éviter que le monde, son oeuvre, ne s'écroulât, fit sacrifier du haut des sept cieux et des sept terres, au moyen de l'éclair, un bélier qu'il créa à la hâte à partir de la matière dense dont est fait le ciel divin, ma kaba. Ce bélier, appelé dyigi makan dyigi, "1'éminent (mouton du) Maître du cie1, l'èminent", ou encore sòn saka (63), "mouton sacrificiel", avait le corps blanc, les extrémités, l'encolure et la tête noires et son front était marquée d'une tache blanche, d'où son troisième nom, doolo saka, "mouton-êtoile", mouton marqué d'une étoile.

(60) Tous les mâ̂tres initiateurs interrogés sur 1'origine de ces animaux gardèrent le silence. Mais aux dires de woloso, descendants d'esclaves, le chien et l'âne seraient aussi nés des éclats de l'arbre des jumeaux primordiaux selon les uns, et selon les autres, ils seraient respectivement sortis des urines de Mousso Koroni et de Pemba, ce qui fait de ces derniers des parents incestueux. Si cela était vrai, il faudrait reconsidérer le statut du chien dans les grands cultes soudanais (il n'y a pas de sacrifice au komo et au Nya, par exemple, sans immolation de chien) et celui de l'âne, victime désignée de 1 'hyène (patron du Komo) et matière oblatoire de choix dans les rites magiques.

(61) Certains contes et légendes bambara présentent les bilisi comme des êtres repoussants à tous égards et dangereux.

(62) Ces actes sont pudiquement appelés kòta, du nom des organes sexuels (kdta signifie en effet "ce que 1 'on détient en propre au bout, derrière ou sous le dos") et ceci pour ne pas employer les mots trop crus qui les dẻsignent.

(63) On appelle également sòn saka, "mouton-Cob de Buffon" (adenoto kob), un mouton qui, comme le gibier son, court parfois en martelant le sol de tous ses sabots en même temps. 


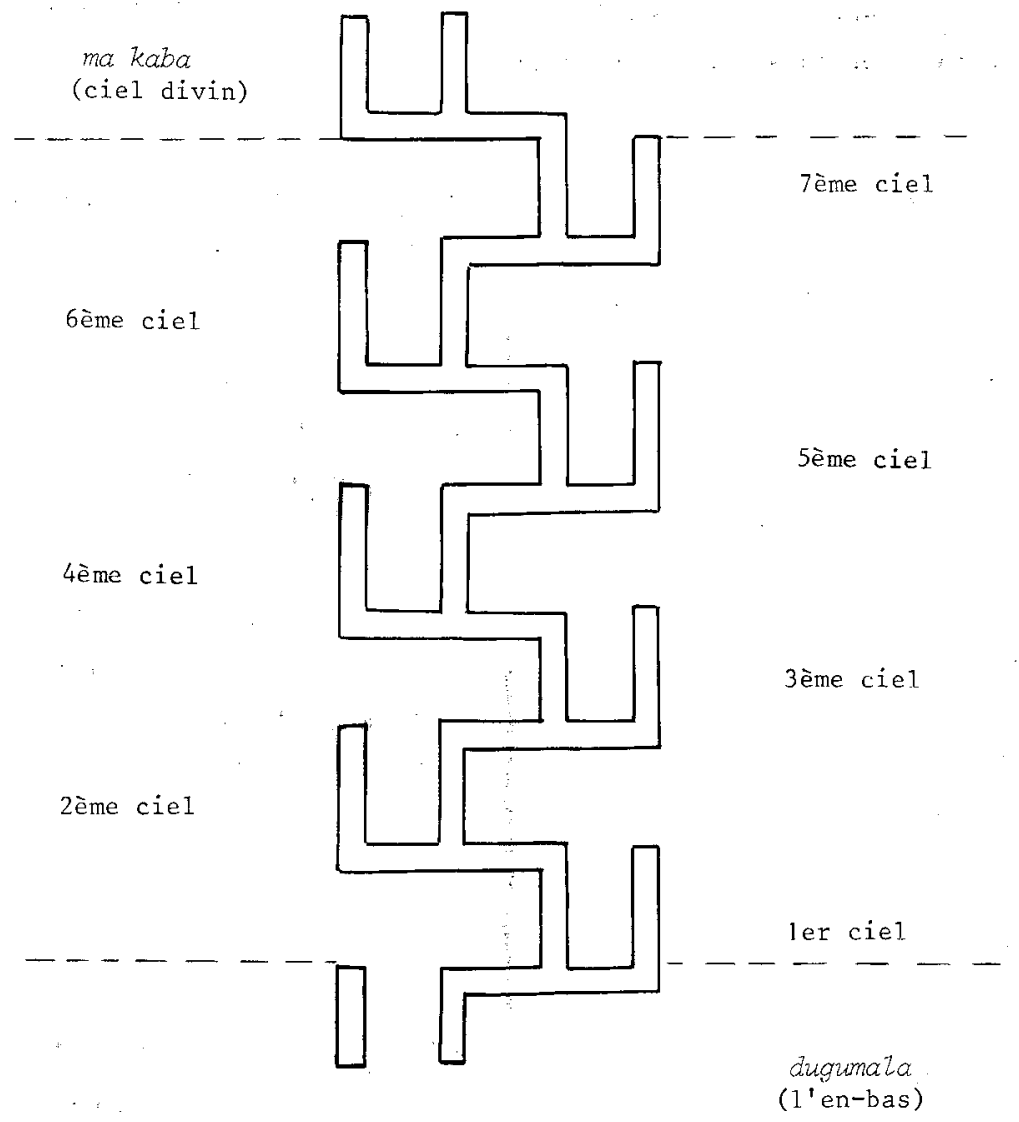

Pl_VIII

Alors que le sang du bélier s'écoulait à travers les dédales de 1 'univers, purifiant et revivifiant țout sur son passage, les morceaux de 1'animal sacrificié étaient quant à eux "léchés" par le cosmos, ce qui eut pour résultat de consolider l'édifice cosmique. 
Le sang de celui-ci s'écoula en suivant les chemins descendants du banan ngoto (64), lavant, purifiant et revivifiant (65) tout l'univers.

Dieu devait ensuite procéder, toujours au moyen de l'éclair, au dépeçage du corps du bélier dyigi makan dyigi dont les quartiers furent répartis comme suit :

1) 1 'épaule et les côtes droites lancées au nord

2) le gigot gauche lancé au sud

3) l'êpaule et les côtes gauches lancées à l'est

4) le gigot droit lancé à l'ouest

5) prẻcipités vers 1 'en-bas et dans l'ordre:

a. l'estomac, les intestins, la rate, le foie, le pancréas, le coeur, les poumons,

b. ensuite, les testicules et le sexe, les reins, la colonne vertébrale et la tête.

En répartissant ainsi les morceaux du bélier sacrifié, Dieu imprima au monde les signes de la femme et de 1 'homme (cf. P1. VII), signifiant pour toujours à ses créatures qu'il fit le monde à l'image de l'homme et pour I'honme et que celui-ci devra à jamais attester de sa suprématie sur toute chose et 1 'adorer en tant qu'"unique Mấtre créateur" sous peine de perdre sa qualité d'home (66).

Bref, dans leur chute à travers 1 'univers, chacun des quartiers du mouton sacrifié tournait comme une toupie (c'est à dire à une très grande vitesse) et faisait vrombir 1 'air ; et lesdits quartiers furent entièrement "léchés", consommés, consumés (67) avant de toucher la

(64) Cf. P1. VIII.

(65) Les mots employés dans le récit bambara sont : ko, laver ; dyo, "lessiver", "laver un objet jusqu'à ce qu'il ne sente plus ni mauvais ni bon"; nyananmaya ko kura, "rendre vivant une nouvelle fois".

(66) Les mythes, légendes et contes bambara et malinké fourmi1lent $d$ 'histoires d'hommes devenus des bêtes ou transformés en rochers à la suite de ruptures d'interdits majeurs.

(67) Nous traduisons par ces mots le vocable nò qui signifie lécher (ses doigts par exemple), user (un instrument de travail qui est comme léché par la matière avec laquelle elle entre en contact). 
terre : c'est ce qui "donna de la moelle à l'univers" (68) et le renforça. Seuls les entrailles enveloppées de graisse et les testicules du bêlier parvinrent intacts à la surface de la terre contre laquelle ils s'écrasèrent, constituant ainsi la première fumure et le premier liquide séminal (69).

Dès qu'il eut, par le sacrifice du bélier, fini de purifier, de fortifier et de rendre "sain", "énergique" 1 'univers, Dieu dépêcha sur la terre un second couple de jumeaux mixtes, Fâro et son jumeau Bemba (70). Ceux-ci descendirent du ciel à bord d'une "pirogue" en or (71) contenant les signes de tous les êtres vivants d'ici-bas, et ils avaient pour mission d'indiquer aux "choses animées" (72) le droit chemin. En effet, le Créateur leur avait ordonné au moment de leur départ du ciel : "Vous direz à mes créatures (73) les paroles que voici:

1) 1 'univers n'a d'autres mâttres que moi, ma ba, le "grand mâtre" ;

2) il n'y a pas d'autres créateurs au monde hormis moi, danbaa ma, le "mâ̂tre créateur" ;

3) i1 n'y a pas d'autres êtres-force, d'autres rois du monde hormis moi, so flaa facma, le "roi des deux mondes" ;

4) il n'y a pas d'autres puissants au monde hormis moi, se tigi, le "dêtenteur du pouvoir" ;

5) vous n'emprunterez d'autre chemin que celui qui conduit vers moi, le chemin vers lequel tous les chemins convergent;

6) vous ne sacrifierez sur d'autres autels que ceux qui auraient êté

(68) ka dunya sèmèntiya. Pour les Malinké et les Banbara, Ia moelle, sèmèn, est la nourriture par excellence des os ; elle sécrèterait "les huiles du corps" dont l'abondance se traduit par la souplesse et la beauté de la peau et du système pileux, et la rareté par le blanchissement des cheveux et le déssèchement de la peau.

(69) nòkò désigne ces deux matières, ainsi que certaines sécrétions gluantes.

(70) Comme Mousso Koroni et Pemba, Dieu créa ces derniers beaux, puissants, intelligents, mais doux. Leurs noms et titres sont les suivants :

-- Fâro : Dyitigui, Mâtigui, Ba-Fâro, Dyamako, Dyoba, Dyomâdyo, etc. ;

-- Bemba : Nya-Makan, Dyinè-Makan, N'Karamoko Sotigui, Dyrimadyo, etc.

(71) Cf. G. Dieterlen et Y. Cissê, op. cit., p. 42.

(72) nin ma fènw, "choses ayant une âme", ici les hommes.

(73) Par créatures, il faut entendre ici les hommes. 
dressés en mon nom ;

7) vous ne yous soumettrez à d'autres mâ̂tres hormis moi, madummantan masa, "le maître sans limite et le mâ̂tre de l'éternité" (74).

A peine Fâro et sa suite s'étaient-elles installées autour de l'arbre dubaten (75) sis au bord de la mare sacrée, koba (76), que Mousso Koroni s'employait avec une énergie redoublêe à tenter de contrecarrer les desseins de Dieu, et ceci en essayant de faire échec à la mission de Fâro. C'est ainsi qu'elle devait dire à l'adresse des nains encore déroutés à la suite de leur déchéance : "Le plus dur pour nous est à présent passé, car rien de pire ne saurait nous arriver ; cela ne doit cependant pas empêcher la vigilance, car les nouveaux venus que sont ces gens feront tout, à n'en pas douter, pour nous subjuguer. Sachons donc nous défendre le moment venu". Se souvenant de ces propos de Dieu : "1'ignorance est obscurité, sinon tout est signe, indice, marque dans ce monde, de même que tout y est (question d') observation" (77), Mousso Koroni devait inviter les nains à partir à la découverte de tout ce qui pouvait contribuer à assurer leur défense. C'est ainsi qu'elle découvrit, avec ses partisans, le secret des plantes (78) et surtout apprit à connâ̂tre les évènements à venir. Voici comment. Le pembete, qui gisait encore à l'endroit où il était tombé du ciel, émettait des sons diffërents selon le temps qu'il faisait, selon l'évènement qui se produisait ou allait se produire, etc.. De plus, sa force, nyama,

(74) 1. tigi wèrè tè dinyè na ne kò, ma ba

2. danbaa wèrè tè dinyè na ne kò, danbaa ma

3. facma wèrè tè dinyè na ne kò, so fla faama

4. sebaa wèrè tè dinyè na ne kò, setigiba

5. ow ti na sira wèrè minè, fo sira min bi taa ne ma, sira bèè kunbèn sira

6. caw ti na dyo wèrè sòn fo ni dyoo min dyòra ne tókò la

7. caw ti na són masa wèrè ye ne kò, ma damantan masa. du de Dieu.

(75) Ficus Punctata. Le mot dubaten signifie "béni", sous-enten-

(76) Cette mare serait formée autour de $1^{\prime}$ abysse qu'aurait forée 1a "pirogue" de Fâro en atterrissant.

(77) donbaliya ye dibi de ye, n'òtè fèn bè ye taamashinyè ye dinyè na, fèn bè ye kòlòshizi ye.

(78) Des plantes vénéneuses, en particulier. 
faisait dêplacer et disposer dans un ordre précis les cailloux qui 1 'entouraient. Mousso Koroni le fit transporter dans un vestibule rocheux et faisait broyer sur lui, pour empêcher son usure, tout être vivant qui s'aventurait dans les lieux (79). De plus, elle chargea 17 nains d'écouter les sons émis par le pembele et d'observer en permanence les figures qui se formaient au fur et à mesure que se déplaçaient les cailloux (80). Ainsi Mousso Koroni et "ses enfants" violèrent-ils le troisième domaine réservé de Dieu en apprenant à lire 1 'avenir dans ces figures. Par la suite, ils faisaient disposer les cailloux par ceux d'entre eux qui entraient en transe (81).

\section{Troisième sacmifice fait par Dieu.}

Quand il apparut à Dieu que la divination liêe au culte du pembele constituait une grave menace pour son messager et les siens, il permit à Ba Fâro, "mère Fâro", de recourir à la force des "grands signes de la création" et à l'observation des étoiles et constellations (82) pour prévoir l'avenir, et ceci dans le double but de prévenir le mal et de faire le bien et rien que le bien...

(79) On dit de cette mise à mort que "Mousso Koroni faisait irrêmédiablement perdre à ces animaux leurs nin ou âmes" (muso kòrò ni tun bè donfèn nunun bònè u nin na).

(80) Selon certaines informations, il aurait existé à l'origine deux sortes de divinations : : -- d'une part, celle liée au pembele, à la terre et aux cailloux, et relevant de Mousso Koroni, des nains et des mangusi (gnomes) ; -- d'autre part, celle liée à l'arche, à l'eau et aux étoiles, et relevant de Fâro, des dyidenw, "enfants de l'eau", et des nyananu, "génies tutélaires" vivant dans les bosquets sacrés. Selon les mêmes informations, la divination par les cailloux et, dans une certaine mesure, la géomancie à 16 cases appartiendraient au premier type, alors que la divination par les cauris et la géomancie à 33 cases, appelée wakataba, seraient du domaine de Fâro. Ces données méritent d'être vérifiées et approfondies.

(81) Nous traiterons des rapports entre la transe et la divination dans notre expose du mythe d'origine de la musique.

(82) Nombreux sont nos informateurs qui insistent sur les rapports étroits existant entre la géomancie (le wakazaba en particulier) et les "signes de la création" et les constellations. Nous espérons pouvoir bientôt expliciter ces rapports. 
Après bien des évènements (83), Mousso Koroni, devenue vieille, bossue et ratatinée, le corps couvert de gerçures et de pustules nauséabondes, les doigts crochus, les yeux congestionnés et larmoyants, les cheveux rares et rêches, Mousso Koroni donc fut tout d'un coup saisie d'un dêsir ardent d'honorabilité et de jeunesse. Et pour cause ! Ne voyait-elle pas chaque jour qui passait apporter plus d'éclat et de frâ̂cheur à la beauté et à la jeunesse de Fâro qui était de surcrô̂t adulée de sa suite! Folle de jalousie, keleya (84), elle s'employa dans un premier temps à pousser les homes issus des signes de l'arche contre leur "guide et protectrice" (85), Fâro. "Regardez-vous d'un peu près, leur lança-t-elle, et vous apercevrez ce que vous êtes devenus, des lambeaux de chair et de vie, et dites-vous bien que vous serez un jour très prochain plus délabrës encore que je ne le suis, alors que celle qui vous trompe en vous narrant de belles histoires et en vous disant de bien douces paroles est plus que jamais éclatante de jeunesse et de beauté".

Nombreuses furent les personnes dites kunangow, "malchanceuses", qui quittèrent alors le camp de Fâro pour celui de Mousso Koroni. Celle-ci parvint également à convaincre les volatiles, pan fènw, "les choses, les êtres qui volent", de la nécessité de se rendre au-delà des cieux afin de s'emparer du secret de "l'éternelle jeunesse" (86). (Dieu n'avait-il pas un jour dit à Mousso Koroni alors qu'elle se trouvait au ciel : "Toute personne que moi, le Mâ̂tre Créateur, gratifierai de 1 'immortalité et du "non vieillissement" saura tout de 1 'univers") (87). Pour ce faire, elle leur avait notamment dit ceci : "En ce qui

(83) Notamment, les pérégrinations et tribulations de Mousso Koroni dont il sera question dans nos prochains travaux. Koroni.

(84) Notons que kele, "jalouse", est l'un des attributs de Mousso

(85) Nous traduisons par ces mots le vocable dèmèbaa ("celle qui aide") qui connote bien 1 a fonction de conductrice, de protectrice, d'éducatrice et de salvatrice de Fâro dans les croyances et dans de nombreux rituels dont ceux du Komo (cf. G. Dieterlen et Y. Cissé, op. cit., p. 250).

(86) kòròbaliya, le "non vieillissement".

(87) ne danbaa ma ma na danfèn fèn shii kè sabaliya ye, ani kòròbaliya, i na dinyè kònò na ko bè dòn. 
vous concerne, vous volatiles, n'oubliez pas que chacun de vous ne dêtient qu'un seul "secret", une seule parcelle du sayoir et une seule. Autant dire que vous serez toujours dans ce monde des aveugles et des sots et, pire encore, des jouets, et ceci quelle que soit votre force, entre les mains des gens de Fâro qui, eux, détiennent déjà individue1lement plusieurs secrets du savoir".

Les volatiles, dont certains étaient d'une taille et d'une puissance inouies -- 1'aigle, le roitelet et le papillon (88), pour ne citer que ceux-1à, réduisaient en poussière tout somet de montagne sur lequel ils se laissaient choir -- crurent volontiers à Mousso Koroni. Ils étaient en route vers Dieu lorsque, au cours d'une halte sur le sol du quatrième ciel, le Créateur fit tomber sur eux un morceau incandescent du cinquième ciel, dit ko bZen kaba, "le ciel des choses rouges", des malheurs (89). Ce fut le plus grand dêsastre que I'univers connût à ce jour. De nombreuses espèces disparurent à jamais, volatilisées ou grillées (90); parmi les survivants, les brûlés graves étaient de loin les plus nombreux. Seuls duga, le vautour, et dugani, la tourterelle, et quelques rares autres volatiles sortirent indemnes du désastre; mais, comme chez leurs semblables, leur taille et leur puissance devaient décrô̂tre considérablement (91).

Cette hécatombe et cette déchéance des volatiles, accompagnées de blessures graves et de changements notables, constituent le troisième sacrifice effectué par Dieu.

Quelques instants après ces ëvènements, le Créateur apostropha en ces termes le forgeron chargé de la surveillance de l'accès au

(88) Les mythes relatifs à ces volatiles sont d'une grande beauté et d'une profondeur philosophique et morale rares. Nous détenons 1 'enregistrement de celui du papillon qui sera reproduit dans notre thèse de doctorat.

(89) Ce ciel serait celui du soleil, kara ba, "le grand cercle" ou "le grand esprit", ou kara blen, "le cercle rouge" ou "1'esprit rouge", symbole de la grandeur et de l'ardeur de "l'esprit divin", kara ma.

(90) On dit que "99999 volatiles sur 100000" périrent ce jour-là.

(91) Selon certaines versions du mythe, tous les volatiles virent à cette occasion décrô̂tre leur taille et leur force; selon d'autres versions, c'est avec la "seconde création", intervenue à la fin du déluge, que les créatures de Dieu devinrent plus petites et moins puissantes. 
cinquième ciel (qu'aucune âme enveloppée dans un corps ne saurait atteindre, à plus forte raison dêpasser) : "Ordonne au vautour de ramasser les morts et de rassembler les blessés afin de les ramener derechef sur la terre, et dis-lui qu'il pourra, ainsi que son compagnon, sumiku, l'hyène, manger la chair des volatiles morts ou ayant des blessures qui saignent ..." C'est depuis ce jour que les êtres vivants se mangent (92). Auparavant, toutes les créatures vivaient de nèèma, "fraîcheur", "douceur" et de maa hinè, "grâce divine", qui entraient en elles par leurs pores et avec I'air qu'elles respiraient (93).

\section{Quatrième sacmifice fait par Dieu.}

Parmi les survivants amenés sur la terre par le vautour figurait bòn, l'aigle, dans le bréchet de qui s'était implanté ce qui restait du fragment de ciel incandescent tombé sur les volatiles. Ce fragment tira jusqu'à les tarir presque complètement le sang et la vigueur de bòn qui restait couché, incapable qu'il était de se dégager de l'emprise de l'aérolithe. Celui-ci, par la volontê de Dieu, se détacha un matin de son corps en y laissant toutefois un petit morceau. Ce dernier tomba à son tour lorsque la blessure de l'oiseau était sur le point de se cicatriser complètement. A cause de leur nature et de leur histoire ( $u k e ̀$ tyoko n'u sònò tyoko) (94), ces deux fragments du ciel sont considérês comme étant les premiers dyoow (95) de la terre,d'où leur nom : dyèn dyo ba fòtò ani dyèn dyo den fòtò,"le premier dyoo mère du monde et le premier dyoo enfant du monde" (sous-entendu des hommes). Et la force qu'ils

(92) dumuni daminèna nyòòn dun de Za, "manger commença par se manger entre gens semblables", dit une boutade des korè duga, les "bouffons sacrés" des Bambara. Ils ajoutent : hali bi, den balo fòlò y'a ba ye, "même aujourd'hui, la première nourriture de 1'enfant est sa mère". Ces propos méritent réflexion.

(93) On croit savoir qu'ils n'éliminaient aucun déchet de quelque nature que ce soit.

(94) Text. : "La manière dont $i 1 s$ sont faits et la manière dont ils furent trouvés", sous-entendu par le forgeron, Noun Fayiri, sous la garde de qui Fâro avait placé le grand blessé du ciel.

(95) On appelle en général dyoo tout objet de culte consacré ; mais en réalité ce terme désigne un autel en pierre, en terre, en fer et en bois, dressé et généralement planté dans la terre. 
dégageaient suffisait, à elle seule, à repousser les ennemis de Fâro et à faire avorter leurs machinations. La mâ̂tresse des eaux, dy tigi (96) les confiera plus tard à Noun Fayiri, le premier forgeron ( de la terre), et ils deviendront ainsi "la première enclume mère du monde et la première enclume enfant du monde" (dyjèn kulan ba fòzò ani dyèn kuzon den fozo).

Rendue plus furieuse que jamais par le don des dyoow fait par Dieu à Fâro et à ses fidèles, Mousso Koroni se mit à crier à tue-tête : "Je ne me soumettrai jamais à qui que ce soit ici-bas, moi Nyakalen Dyatara (97), même s'il faut pour cela que tous les êtres disparaissent de la surface de la terre. Oui, je ne serai point la "domestique" (98) de personne" (99).

Effrayés par ces imprécations dont ils mesuraient d'autant mieux la gravité qu'ils avaient souvent entendu Mousso Koroni évoquer avec effroi, elle qui n'avait jamais peur de rien, 1'éventualité d'un second cataclysme pour le monde, les nains, sans bruit, se mirent à aménager, en le consolidant et en le colmatant comme il faut, 1'intérieur d'une grotte attenante à 1'auvent dans lequel gisait le penbele.

Quant à Mousso Koroni, elle ne tarda pas à passer aux actes. Usant de toutes sortes de maquillages (100) pour se rendre sinon belle, du moins attirante ou désirable, elle attirait tous les jours dans sa

(96) Cf. note 70

(97) "Nyakalen à 1'esprit ardent et sans peur".

(98) bolotamoko, "personne (qui est) dans la main" (au service exclusif d'une autre personne).

(99) Selon mon mâ̂tre et ami, le vieil esclave Bâ-Molobali Dembélé, "Mousso Koroni, durant toute sa traversée du monde, employa toutes les ressources de son être à la conquête et à la sauvegarde de trois choses : dire ce que lui dictait sa volonté ; faire ce que lui dictait son désir ; être propriétaire d'elle-même (être libre de tout lien de dépendance)". (muso kòròni y'a ka dinyè latikè bèn ko saba de ma : sako na kuma fò, dunkò na kokè, ani kè n'yèrè ye ni kè n'yèrè ta ye). Et notre interlocuteur de faire cette réflexion : "1'esclavage n'est pas une bonne chose et toute personne au cou de qui on aura placé la corde de 1'esclavage perđra à jamais sa qualité d'homme" (dyònriya ma nyi, n'i dyònnya dyum bizaza $i$ kan na ka ban, o y'e bòzen ye mòkòinfinmya na).

(100) Elle avait surtout recours aux couleurs chaudes, vives, et s'était confectionné une perruque aux innombrables tresses, appelée tyè gosi, "frapper les hommes" (autrement dit, les séduire). 
tanière un nombre de plus en plus élevé de jeunes gens du camp de Fâro, qui devenaient, mais pour quelques instants seulement, ses amants; car son nyama tuait en très peu de temps ceux qui 1'approchaient, a fortiom la pénétraient. Elle jetait aussitôt le corps de ses victimes à ses chiens qui les dévoraient entièrement (101). Néanmoins, Mousso Koroni devait tomber enceinte (102) des oeuvres d'un de ses "petitsfils" (103).

Enfin ce qui devait arriver arriva, d'autant que Dieu ne se dédit point. Pendant sept jours, au cours desquels le soleil se maintint au zénith, dardant de tous ses rayons, 1'eau n'arrêta pas de sourdre de 1'abysse creusêe par la chute de la "pirogue de Fâro" ; puis pendant sept nuits, au cours desquelles le soleil resta au nadir, favorisant. la descente sur la terre d'un froid glacial, une pluie torrentielle ne cessa de tomber du ciel sillonné d'éclairs et tremblant de coups de tonnerre.

C'est au cours de ce "grand jaillissement" (sous-entendu, de l'eau de la terre et de l'eau du ciel), funti ba, qui submergea la terre toute entière, que périrent tous les "enfants" de Mousso Koroni (104), à I'exception des nains qui parvinrent à s'emmurer dans leur grotte. Mousso Koroni elle-même devait à son tour, après avoir eu les yeux grillés par deux rayons de soleil (105) et I'utérus (et ce qu'il

(101) Sur l'origine du chien, voir note 60.

(102) Il s'agissait, dit-on, d'une grossesse nerveuse que les Bambara et les Malinké appellent dyinè kónò, "grossesse (provoquée par) des génies".

(103) Au sens classificatoire, s'entend. En effet, la première génération de personnes créées par Dieu est représentée par Mousso Koroni et Fâro et leurs jumeaux respectifs ; la seconde par les géants devenus les nains; la troisième par les gens issus de l'arche de Fâro.

(104) muso kòròni denw, c'est à dire tous ceux qui avaient épousé la cause de Mousso Koroni.

(105) Tout être en mourant emporte dans les yeux 1'image du dernier regard qu'il aura jeté sur le monde. La fixation de cette image est concomitante au départ du "petit double intelligent", dya nin kekun, lequel quitte le corps par les pupilles. En faisant griller les yeux de Mousso Koroni, Dieu non seulement détruisit toute trace de 1 'univers dans son corps, mais anéantit aussi en elle toute parcelle de "raison". 
contenait) arraché, puis avalé par le poisson sumè (106), mourir noyée et transie de froid, dans la solitude la plus complète. Son corps fut entièrement dissous dans les eaux du déluge. Elle apparâ̂t de nos jours encore -- et il en sera ainsi jusqu'à la fin des temps -- chez tous les êtres animés et particulièrement chez la personne humaine, sous forme de tere dyugu (107) : mauvais penchants, difformités, tares physiques, guigne, poisse, déviations de tous genres, etc. (108).

Quant à Fâro enfin, elle sauva sans aucune difficulté tous ses fidèles de la mort, qu'ils fussent humains ou animaux, et ceci grâce à sa"pirogue" à la poupe (109) de laquelle elle se tenait, armée de sa pagaie, dès le début du dêluge : tout être qui touchait la proue de ladite pirogue était aussitôt transformé en son signe d'origine et tombait dans l'embarcation.

7. Le début de l'ère de Fâro.

A la fin du retrait des eaux du dêluge, dont le début a été marqué par le lever simultané de sigi doolo (Sirius) et du soleil (110), Fâro sema à la volée -- tout comme après sa descente du ciel -- les signes des êtres animés (homme, animaux, plantes). Ces signes, en

(106) Poisson des profondeurs dont les os, très recherchés des sorciers, contiennent du poison, ce qui rend leurs piqûres mortelles.

(107) A propos de la notion de tere, cf. G. Dieterlen, Essai sur Za religion bambara, Paris, PUF, 1951, pp. 61-63 et Y. Cissé in La notion de personne ..., op. cit., pp. 158-160.

(108) Les eaux du déluge ayant submergé la terre toute entière, tout être né sur cette terre après le déluge et consommant de I'eau véhicule une partie de ce tere de Mousso Koroni.

(109) C'est par les mots dyuu ("fondement") et kuun (tête) ou nèèn ("langue") que les Malinké et les Bambara désignent respectivement la poupe et la proue de 1 a pirogue.

(110) Cet évènement mythique, qui traduit par ailleurs une réalité astronomique, est matérialisé par un masque dédié à Fâro chez les Bambara et par une peinture rupestre chez les Malinké. 
buvant l'eau du sol et en mangeant la terre noire de la mare de Fâro, donnèrent naissance à la vie actuelle (111).

Ainsi naquit le "soleil", 1'ère de Fâro. Celle-ci forma alors au plus profond d'elle-même le voeu (112) de "sanctifier" (saraka) (113), de rendre prospère son règne et se demanda ce qui ferait le plus plaisir au créateur. En réponse à ce voeu, Dieu ressuscita sur le champ et fit sortir des eaux de la mare de Fâro dyigi makan dyigi, le bélier qu'il avait été amené à créer et à sacrifier au ciel pour sauver le monde d'un effondrement certain (114). Et Fâro fit sacrifier ce bélier après qu'il eut couvert les neufs brebis primordiales (115).

C'est depuis lors, dit-on, que tout sacrifice vrai suppose la rêsurrection de la victime (116).

(111) Les Bambara et les Malinké classent les "choses créées", dan fènw, en quatre groupes ou générations : a) les êtres créés au ciel ; b) les gêants et leurs avatars, les nains ; c) les êtres nés avant le déluge, issus des signes que Fâro transporta du ciel sur 1a terre à bord de sa "pirogue"; d) les êtres créés après le déluge.

(112) Nous traduisons par voeu le mot naniya qui désigne chez l'être humain la composante spirituelle d'où naissent les bonnes comme les mauvaises intentions.

(113) C'est à ce vocable, et non au mot sòn ("offrir", "arroser", "sacrifier"), que les Bambara ont recours lorsqu'ils sanctifient un objet ou veulent rendre prospère un pays, un règne, etc.

(114) Cf. supra, p. 47.

(115) Les moutons à tête et à encolure noires seraient les "manifestations", Zaboden, du bélier primordial. En revanche, celui-ci aurait pour "répondants", lasigi, les béliers aux cornes d'or vivant au fond des puits sacrés en compagnie du serpent ninkin-nankon, dispensateur de pluies et de richesses.

(116) Nous verrons, dans la suite de cet article, ce qu'il en est exactement. 
Cette première partie du mythe d'origine du sacrifice bambaramalinké appelle quelques observations.

Notons tout d'abord le lien privilégiê existant, d'une part, entre la faute (blasphème, transgression, rupture d'interdit) et la mise à mort-sanction, êlevée au rang de sacrifice dans le mythe et, d'autre part, entre le sacrifice dit vrai et la restauration de l'édifice cosmique, dégradé précisément à la suite de la faute ci-dessus mentionnëe.

Notons aussi que le sacrifice dit vrai fut le prélude à la naissance d'une nouvelle humanité, de même que le déluge marqua à la fois la fin d'une ère de heurts et de malheurs et le début de l'ère du bonheur de vivre.

Aucune des innombrables victimes de trois des quatre "sacrifices primordiaux" -- ces trois sacrifices ayant été de violentes mises à mort accompagnées d'effroyables mutilations et blessures, de déformations et de déchéances inoules chez les sujets qui échappèrent à la mort -- ne ressuscita ou ne réapparut à la vie sous une forme ou une autre. Par contre, dyigi makan dyigi, le bélier céleste sacrifié par Dieu émergea, après le déluge, des profondeurs de la mare de Fâro. C'ést bien là l'une des caractéristiques essentielles du "vrai et bon sacrifice" dans lequel la victime ou ses morceaux judicieusement répartis ne sont, par ailleurs, jamais réduits à néant, mais servent d'abord et avant tout à restaurer, à consolider ou à fertiliser tout ou partie de 1 'univers.

Une autre caractéristique essentielle du vrai sacrifice est la place de la marque et du signe tant chez la victime sacrificielle que dans la répartition de ses morceaux après la mise à mort.

Enfin le vrai sacrifice suppose 1a "consommation", quelles qu'en soient les modalités, d'une partie ou de la totalité de la victime sacrificielle.

(A suivre) 